\title{
Long-term surgical outcomes of multiple parfoveolar curvilinear internal limiting membrane peeling for myopic foveoschisis
}

\author{
Tian Tian ${ }^{1} \cdot$ Haiying Jin ${ }^{1} \cdot$ Qi Zhang ${ }^{1} \cdot$ Xiang Zhang $^{1} \cdot{\text { Hongtao } \text { Zhang }^{2} \cdot \text { Peiquan Zhao }}^{1}$ \\ Received: 19 December 2017 / Revised: 18 June 2018 / Accepted: 22 June 2018 / Published online: 30 July 2018 \\ (c) The Royal College of Ophthalmologists 2018
}

\begin{abstract}
Purpose To investigate the long-term results of a modified technique for parafoveal multiple curvilinear internal limiting membrane (ILM) peeling to preserve the epi-foveal ILM in myopic foveoschisis surgery.

Methods Thirty-two consecutive patients (36 eyes) were retrospectively reviewed. Patients were divided into two groups according to the extent of ILM peeled: the fovea-sparing ILM peeling group (FS) (18 eyes) and total ILM peeling group (TP) (18 eyes). Patients were followed up for at least 12 months. The main outcome measures were best-corrected visual acuity changes, evolution of macular schisis and the factors associated with the development of a full-thickness macular hole (FTMH).

Results FTMH developed in 1 of 18 eyes $(5.6 \%)$ in the FS group and 3 of 18 eyes $(16.7 \%)$ in the TP group $(P=0.28)$. Long-term follow-up showed visual improvement was better in the FS group than in the TP group (0.94 vs. 0.58 logMAR). Macular schisis disappeared in 13 of 18 eyes (72.2\%) in the FS group, but disappeared in 7 of 18 eyes (38.9\%) in the TP group $(P=0.04)$. Logistic regression analysis showed that only the preoperative outer lamellar macular hole $(P=0.016)$ was a significant risk factor for development of postoperative FTMH.

Conclusions Fovea-sparing ILM peeling achieves a higher rate of macular schisis resolution over total peeling. A preoperative outer lamellar macular hole can be a risk factor for the development of a macular hole.
\end{abstract}

\section{Introduction}

Myopic foveoschisis is a unique complication of high myopic eyes and a major cause of visual impairment. The pathogenesis of myopic foveoschisis is varied; tangential vitreous traction of the disease is believed to be the primary cause [1-3]. Presently, vitrectomy and internal limiting membrane (ILM) peeling are considered to be effective in the management of high myopic foveoschisis [4-6].

Electronic supplementary material The online version of this article (https://doi.org/10.1038/s41433-018-0178-0) contains supplementary material, which is available to authorized users.

Peiquan Zhao

zhaopeiquan@xinhuamed.com.cn

1 Department of Ophthalmology, Xinhua Hospital, Affiliated to Medicine School of Shanghai Jiao Tong University, Shanghai, China

2 Department of Ophthalmology, Bin Zhou Hubin Aier Eye Hospital, Shandong, China
However, it has been reported that the risk of developing a full-thickness macular hole (FTMH) ranges from 16.7 to $20.8 \%$ in myopic foveoschisis eyes undergoing ILM peeling $[7,8]$. The mechanisms of why and how FTMHs develop postoperatively have not been fully determined. Hirakata and associates reported that the risk of postoperative FTMH was higher in eyes with foveal retinal detachment (RD). Recently, Shimada and associates hypothesized that ILM peeling on such a thinned central foveola could induce a break in the central foveolar tissue, leading to the formation of FTMH.

The development of FTMH is a serious complication in highly myopic eyes [2, 3, 7-14]. To address this, Ho et al. [15] and Shimada [7] proposed preserving the epi-foveal ILM during ILM peeling to prevent the formation of FTMH after vitrectomy. They reported that this new technique achieved better anatomical and visual results over total ILM peeling, consequently preventing long-term foveal retinal thinning and the development of FTMH [7, 9, 15]. Nevertheless, the surgical manoeuver to preserve the epifoveal ILM is not a simple manipulation, especially for highly myopic eyes with foveal RD. Moreover, although the 
Fig. 1 Schematic illustrations of the parafoveal multiple curvilinear internal limiting membrane (ILM) peeling technique. a ILM peeling was initiated and centred away from the central fovea in a continuous curvilinear manner in each quadrant, nasal, temporal, inferior and superior. b An epifoveal ILM of about $500 \mu \mathrm{m}$ in diameter was preserved after removing the residual ILM between the four circular areas
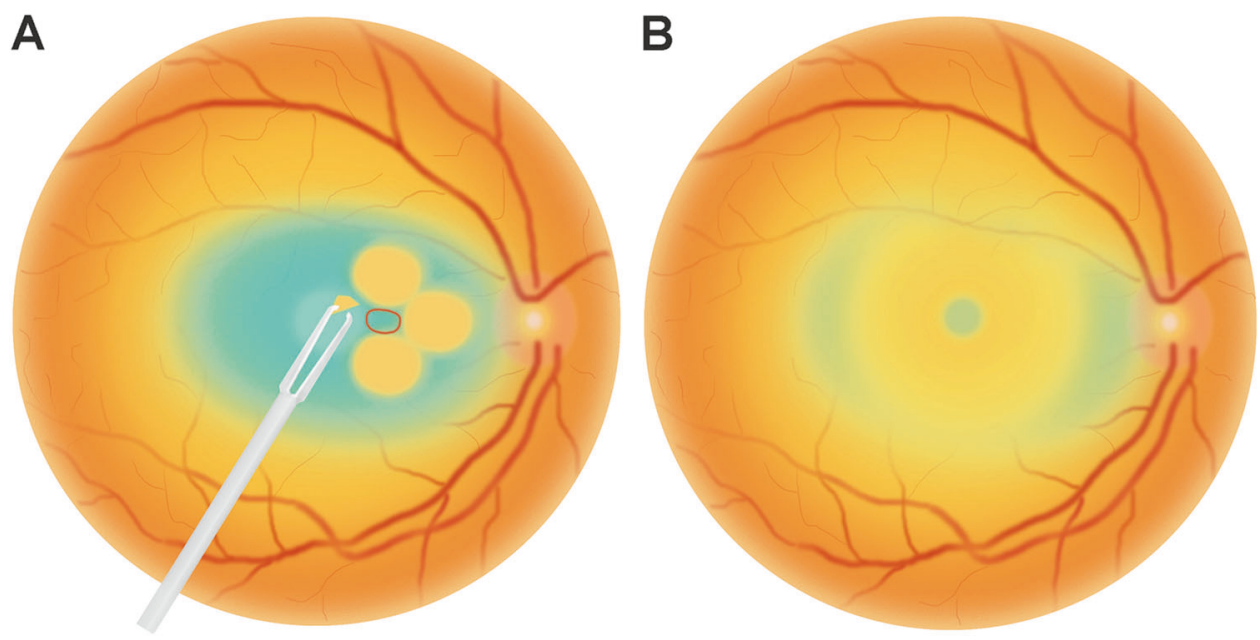

fovea-sparing ILM peeling technique has been evaluated, further studies in multiple centres are necessary to fully determine the effectiveness and safety of this procedure.

In the current study, we described the long-term surgical outcomes of this modified fovea-sparing ILM peeling technique for a large number of patients with a long follow-up.

\section{Subjects and methods}

\section{Subjects}

This study adhered to the tenets of the Declaration of Helsinki and was approved by the institutional review board of the Xinhua hospital-affiliated medical college, Shanghai Jiao Tong University. All participants provided written informed consent of the possible benefits and risks. This study was a retrospective, interventional and consecutive case series of myopic foveoschisis patients who underwent vitrectomy with fovea-sparing or total ILM peeling from January 2014 to June 2016. The inclusion criteria were a preoperative spherical equivalent higher than -8.00 dioptres (D) or axial length (AL) longer than $26 \mathrm{~mm}$ and progressive visual loss presumably caused by foveoschisis associated with high myopia. Eyes with dense opacities of the media such as corneal opacities or dense cataracts were excluded. The exclusion criteria also included a preoperative FTMH observed by spectral-domain optical coherence tomography (OCT), poor visual acuity (VA) due to diffuse macular chorioretinal atrophy or a large Fuchs spot, high myopic choroidal neovascularization, a history of ocular trauma, and other systemic and retinal diseases that may affect VA.

Patients were divided into two groups according to the ILM peeling procedure: Group 1: fovea-sparing ILM peeling group (FS) (18 eyes) and Group 2: total ILM peeling group (TP) (18 eyes). All patients underwent a complete ocular examination, including a slit-lamp examination, best-corrected VA (BCVA) measurement, fundus examination and OCT (RTVue-100, Optovue, Inc., Fremont, CA, USA) scanning at every visit. The AL was measured using an optical biometer (Ver 5.4.Carl Zeiss Meditec AG, Jena, Germany). All patients were followed up for at least 12 months.

\section{Surgical procedure}

Phacoemulsification with simultaneous intraocular lens implantation followed by a standard 23-gauge three-port transconjunctival pars plana vitrectomy was performed on all eyes in the two groups under local anaesthesia. First, the central vitreous core was removed, and then the posterior hyaloid was removed by active suction using a vitreous cutter. Then, the ILM was stained with brilliant blue $\mathrm{G}$ for $30 \mathrm{~s}$ in all patients. In the FS group, the macular area was divided into four quadrants: the nasal, temporal, superior and inferior regions. The initial ILM tear was performed to make a flap away from the central fovea in one quadrant using a microforceps (Grieshaber, Alcon, Fort Worth, TX, USA). Then, the ILM was peeled in a curvilinear manner centred around the site away from the central fovea in each quadrant. Following parafoveal curvilinear ILM peeling, small areas of residual ILM between the four circular areas of ILM were removed. The edge of the residual ILM at the central fovea was trimmed by a vitreous cutter. After multiple parafoveal curvilinear ILM peeling, an epi-foveal ILM of approximately $500 \mu \mathrm{m}$ in diameter was preserved [16]. (Fig. 1) In the TP group, the ILM was peeled over the complete macula with an approximately two-disc diameter area. Finally, fluid-air exchange was performed, followed by an injection of $16 \%$ perfluoropropane. 


\section{Statistical analysis}

Statistical analysis was performed using the Sigma Stat software (SPSS, Inc., Chicago, IL, USA) for Windows software version 19.0. The age, AL, preoperative and postoperative BCVA (logarithm of the minimal angle of resolution, logMAR); visual improvement in logMAR; and preoperative and postoperative central foveolar thickness (CFT) were compared using the Mann-Whitney $U$ test. Differences in the incidence rates of the outer lamella macular hole, foveal detachment and evolution of macular schisis between two groups were analysed using Fisher's exact test. Logistic regression models were used to determine the influence of the potential factor on the development of postoperative macular holes. All $P$ values were two-sided, with $P<0.05$ being considered statistically significant.

\section{Results}

\section{Baseline characteristics of patients}

Thirty-two consecutive patients (36 eyes) who underwent high myopic foveoschisis surgery by one surgeon (PQZ) were enroled and retrospectively reviewed in this study. The baseline characteristics of the 36 eyes and 32 patients are shown in Table 1 . The mean age was $52.8 \pm 10.8$ years in the FS group and $58.0 \pm 13.2$ years in the TP group $(P=$ $0.10)$. The mean AL was $30 \pm 1.7 \mathrm{~mm}$ in the FS group and $28.9 \pm 2.6 \mathrm{~mm}$ in the TP group $(P=0.32)$. The mean refractive error was $13.5 \pm 1.6$ dioptric spherical equivalents in the FS group and $12.8 \pm 2.3$ dioptric spherical equivalents in the TP group $(P=0.31)$. The outer lamellar macular hole (OLMH) was observed in 2 of 18 patients $(11.1 \%)$ in the fovea-sparing group and 1 of $18(5.6 \%)$ patients in the total

Table 1 Baseline characteristics of the patients who underwent foveasparing or total internal limiting membrane peeling

\begin{tabular}{llll}
\hline & $\begin{array}{l}\text { FS group (18 } \\
\text { eyes) }\end{array}$ & $\begin{array}{l}\text { TP group (18 } \\
\text { eyes) }\end{array}$ & $P$ value \\
\hline Age (years), mean \pm SD & $52.8 \pm 10.8$ & $58.0 \pm 13.2$ & 0.10 \\
Sex (female/male) & $11 / 7$ & $10 / 8$ & 0.73 \\
AL (mm), mean \pm SD & $30 \pm 1.7$ & $28.9 \pm 2.6$ & 0.32 \\
RE (D), mean \pm SD & $13.5 \pm 1.6$ & $12.8 \pm 2.3$ & 0.31 \\
OLMH, no. (\%) & $2(11.1 \%)$ & $1(5.6 \%)$ & 1.00 \\
FD, no. (\%) & $10(55.6 \%)$ & $9(50 \%)$ & 1.00 \\
Follow-up (months), & $20.9 \pm 9.6$ & $19.6 \pm 4.1$ & 0.94 \\
mean \pm SD & & & \\
\hline
\end{tabular}

$F S$ fovea-sparing, $T P$ total peeling, $A L$ axial length, $R E$ refractive error, $D$ dioptres, $S D$ standard deviation, $O L M H$ outer lamellar macular hole, $F D$ foveal retinal detachment peeling group $(P=1.00)$. The foveal detachment was observed in 10 of $18(55.6 \%)$ patients in the fovea-sparing group and 9 of 18 (50\%) patients in the total peeling group $(P=1.00)$. The mean follow-up time was $20.9 \pm 9.6$ months in the FS group and $19.6 \pm 4.1$ in the TP group $(P=0.94)$. The differences in these demographic and baseline characteristics were comparable and not significant.

\section{Visual outcomes}

There were no significant differences in the preoperative and postoperative BCVA between the two groups (Table 2). Compared to the preoperative BCVA, the postoperative BCVA improved significantly both in the FS group and the TP group $(P<0.001$ vs. 0.04). The visual improvement, however, was larger in the FS group $(0.94 \pm 0.8 \log$ MAR $)$ than that in the TP group $(0.58 \pm 0.5 \log$ MAR $)$, although this was not statistically significant $(P=0.17)$.

In the FS group, VA improved by more than two lines in all eyes except one eye that developed FTMH, in which case, VA decreased from $0.4 \log$ MAR to $1.0 \log$ MAR. In the TP group, nine eyes (50\%) had a more than two lines of VA improvement. However, BCVA was reduced in four eyes $(22.2 \%)$ in the TP group, and three of the four eyes developed FTMH postoperatively.

\section{Anatomic outcomes}

Postoperative CFT decreased significantly both in two groups $(P<0.001)$. At the final follow-up, macular schisis disappeared in 13 eyes (72.2\%) in the FS group and 7 eyes (38.9\%) in the TP group $(P=0.04)$. In the FS group, the preserved ILM was observed in 2 of 18 eyes (Figs. 2 and 3).

Long-term follow-up showed that FTMH developed in 1 of 18 eyes (5.6\%) in the FS group and in 3 of 18 eyes $(16.7 \%)$ in the TP group postoperatively $(P=0.28)$. The details of the patients who developed FTMH postoperatively are shown in Supplementary Table 1. For the one eye that developed FTMH in the FS group, OLMH was detected by OCT scanning preoperatively (Fig. 2). For the three eyes that developed FTMH in the TP group, OLMH was observed in one eye and foveal RD was observed in the remaining two eyes. These three patients in the TP group received a secondary operation; the MHs were closed and BCVA improved in all three eyes (Fig. 3).

No significant differences in $\operatorname{AL}(P=0.680)$, extent of schisis $(P=0.213)$, presence of posterior staphyloma $(P=$ 0.566), presence of foveal detachment $(P=1.000)$, or ILM peeling type $(P=0.566)$ were detected between patients with and without postoperative FTMH. Among the characteristics examined in the OCT images, the height of schisis $(P=0.028)$ and the percentage of eyes with OLMH $(P=0.027)$ were statistically significantly different between 
Table 2 Visual and anatomic outcomes of before and after surgery in fovea-sparing and total peeling group

\begin{tabular}{llll}
\hline & FS group (18 eyes) & TP group (18 eyes) & $P$ value \\
\hline Preoperative BCVA in logMAR, mean \pm SD & $1.46 \pm 0.8^{\mathrm{a}}$ & $1.11 \pm 0.8^{\mathrm{b}}$ & $0.18^{\mathrm{c}}$ \\
Postoperative BCVA in logMAR, mean \pm SD & $0.56 \pm 0.3^{\mathrm{a}}$ & $0.67 \pm 0.5^{\mathrm{b}}$ & $0.33^{\mathrm{c}}$ \\
Visual improvement in logMAR, mean $\pm \mathrm{SD}$ & $0.94 \pm 0.8$ & $0.58 \pm 0.5$ & $0.17^{\mathrm{c}}$ \\
Preoperative CFT (mm), mean \pm SD & $615.17 \pm 169.7^{\mathrm{d}}$ & $631.6 \pm 146.3^{\mathrm{e}}$ & $0.28^{\mathrm{c}}$ \\
Postoperative CFT (mm), mean $\pm \mathrm{SD}$ & $143.36 \pm 52.4^{\mathrm{d}}$ & $141.6 \pm 93.4^{\mathrm{e}}$ & $0.92^{\mathrm{c}}$ \\
Evolution of MS, eyes no. $(\%)$ & & & $0.04^{\mathrm{f}}$ \\
Disappeared & $13(72.2 \%)$ & $7(38.9 \%)$ & \\
Improved & $5(27.8 \%)$ & $11(61.1 \%)$ & \\
Postoperative FTMH & $1(5.6 \%)$ & $3(16.7 \%)$ & $0.28^{\mathrm{f}}$ \\
\hline
\end{tabular}

$B C V A$ best-corrected visual acuity, $F S$ fovea-sparing, $T P$ total peeling, FTMH full-thickness macular hole, $C F T$ central foveal thickness, $S D$ standard deviation

${ }^{a} P<0.001$ (Mann-Whitney $U$ test)

${ }^{\mathrm{b}} P=0.04$ (Mann-Whitney $U$ test)

${ }^{\mathrm{c}}$ Mann-Whitney $U$ test.

${ }^{\mathrm{d}} P<0.001$ (Mann-Whitney $U$ test)

${ }^{\mathrm{e}} P<0.001$ (Mann-Whitney $U$ test)

${ }^{\mathrm{f}} P$ : Fisher's exact test. patients with and without postoperative FTMH (Supplementary Table 2). Logistic regression analysis with a stepwise method showed that among all of the relevant factors, only the percentage of OLMH was significantly correlated $(P=0.016)$ with the development of postoperative macular holes.

\section{Discussion}

By comparing the long-term surgical outcomes of foveasparing and total ILM peeling for high myopic foveal schisis, this study showed that the rate of thorough resolution of foveolar schisis was higher in the FS group than in the TP group. BCVA improved significantly both in the FS group and the TP group. The visual recovery, however, was better in the FS group than in the TP group. At the last follow-up, there was no significant difference in CFT between the two groups. Preoperative OLMH can be a risk factor for the development of postoperative FTMH.

Recently, Shimada et al. [7] and Ho et al. [14] proposed the epi-foveal ILM preservation during ILM peeling. The technique used in the Shimada's study [7] was named 'fovea-sparing ILM peeling', which started away from the central fovea and restarted from a new site when the peeled ILM flap came close to the central fovea. In the Ho's study [15], the co-authors used microscissors to make multiple tangential cuts around the fovea during the procedure (named as 'foveola nonpeeling technique'). By contrast, Zhao's technique [16], named 'fovea-sparing ILM peeling using multiple parafoveolar curvilinear peels', utilized peeling that was initiated and centred away from the region of the ILM, where it can hopefully remain undisturbed, instead of centring around the fovea. Since ILM peeling was initiated and centred away from the central fovea, it reduced the lift of the ILM overlying the foveal centre and left it undisturbed. The preserved epi-foveal ILM can be trimmed by a vitreous cutter and is left with a sharp ILM margin.

Until now, there has been no consensus on the size of the preserved ILM. In our study, the size of the preserved ILM was approximately 500 to $1000 \mu \mathrm{m}$. In the Ho's study [15], the size of the preserved ILM was 300 to $500 \mu \mathrm{m}$. However, in Shimada's study [7], the size of the spared-fovea ILM was approximately equal to the size of the optic disc. As tangential vitreous traction is believed to be a main factor in the development of myopic schisis, the preserved ILM should be small enough to release all of the traction around the fovea. Additionally, if the size is not small enough or the margin is not sharp, the preserved ILM itself may postoperatively cause contraction. On the other hand, the inner retina will follow a larger arc along with the reattachment of the retina, resulting in the preserved ILM enlargement especially in cases with staphyloma. This factor should also be considered during the ILM preserving procedure. In Shimada's study [7], remaining ILM contraction was observed in 10 of 15 eyes 3 months after the fovea-sparing ILM peeling. The ILM contraction was not found in any cases in Ho's study [9] at the last follow-up. In this study, a preserved ILM was observed in 2 of 18 eyes in the FS group, but no parafoveolar elevation was caused by the contraction of the preserved ILM. Regarding the foveal structure, the fovea itself is a $1500 \mu \mathrm{m}$ depression in the centre of the macula, and the foveola is approximately $350 \mu \mathrm{m}$ in diameter. Thus, $500 \mu \mathrm{m}$ may be the ideal size of 
Fig. 2 Two patients who underwent fovea-sparing internal limiting membrane (ILM) peeling. a Preoperative optical coherence tomography (OCT) image of a 30-year-old male with an axial length of $30.84 \mathrm{~mm}$. Vitreous macular traction and the outer lamella hole (OLMH) (red arrow) with macular retinoschisis were observed in OCT image. The preoperative best-corrected visual acuity (BCVA) was 0.4 logMAR. b At 1 month after surgery, a full-thickness macular hole (FTMH) developed and the BCVA decreased to 1.0 logMAR. The preserved ILM was torn and the rolled margin was detected (yellow arrow). c Preoperative OCT image of a 65-year-old female with an axial length of $28.62 \mathrm{~mm}$. The preoperative BCVA was 1.2 $\log$ MAR. The macular schisis with foveal retinal detachment (RD) (asterisk) was observed. d At 3 months after surgery, the retinoschisis was decreased with an enlarged foveal RD (asterisk). e At 12 months after surgery, the macular retinoschisis was resolved with no contraction of the preserved ILM

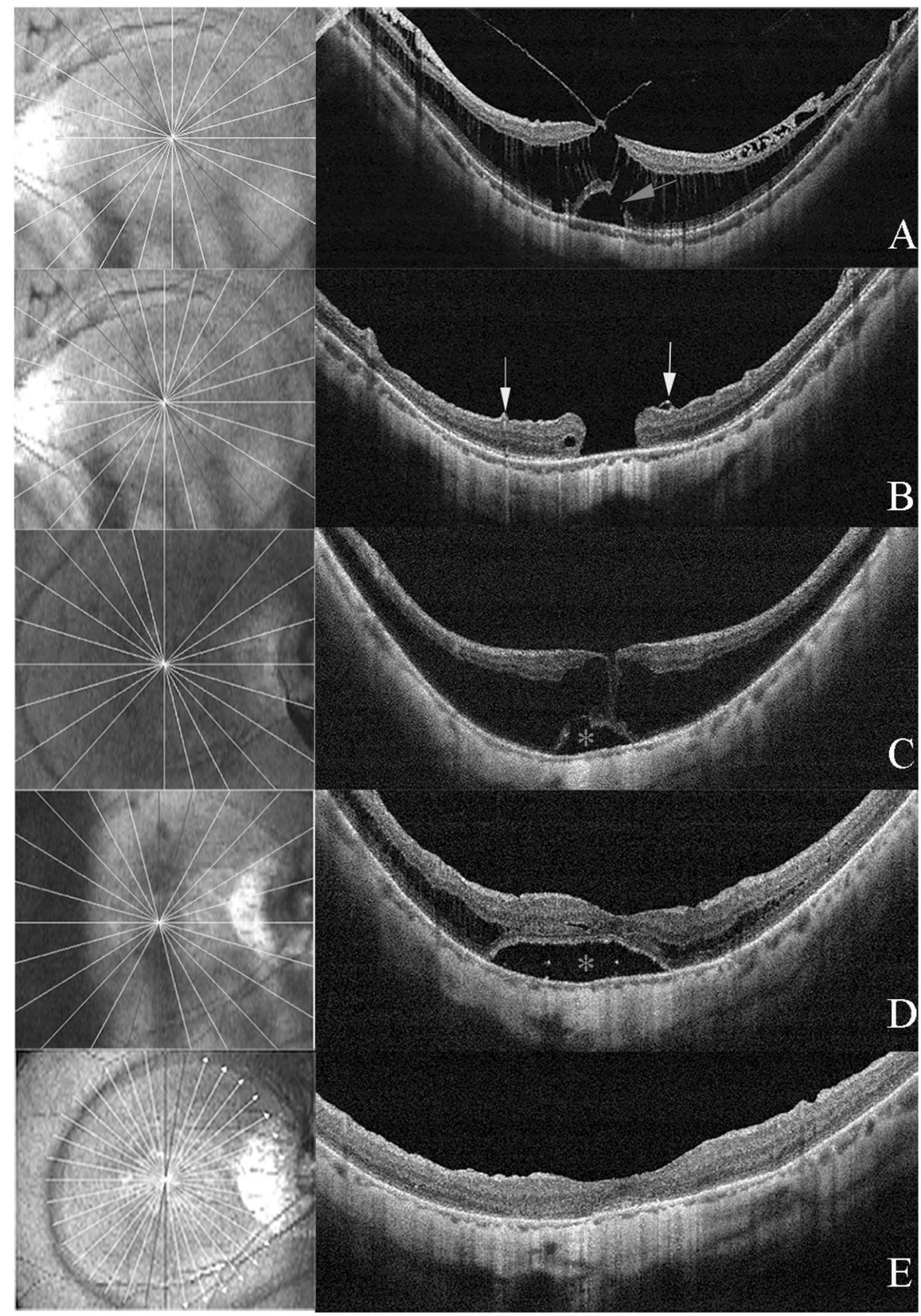

the preserved ILM size, as it can release tangential traction as well as offer enough area to cover the foveola to prevent the formation of postoperative FTMH.

In Shimada's [7] and Ho's [9] studies, no postoperative development of FTMH was found in the fovea-sparing ILM peeling group. The rate of postoperative FTMH in the TP was $16.7 \%$ in Shimada's study [7], 28.9\% in Ho's study [9] and $16.7 \%$ in our study. In our study, however, 1 of 18 eyes (5.6\%) in the FS group developed FTMH within 1 month after operation. In this case, the OLMH was detected during baseline OCT scanning. Analogously, OLMH was observed in 1 of 3 eyes that developed postoperative FTMH in the TP group. The remaining two eyes were accompanied by foveal $\mathrm{RD}$. It has been reported that foveal RD and preoperative inner segment/outer segment junction defects are risk factors for the development of postoperative FTMH in myopic macular schisis [17, 18]. In the current study, logistic regression analysis showed that the presence of OLMH was correlated significantly correlated with the development of postoperative FTMH. The mechanism of why and how FTMH developed in these cases was not fully determined. We hypothesized that the foveal tissue with OLMH is extremely weak and can be more easily damaged, regardless of whether it is managed with fovea-sparing or total ILM 


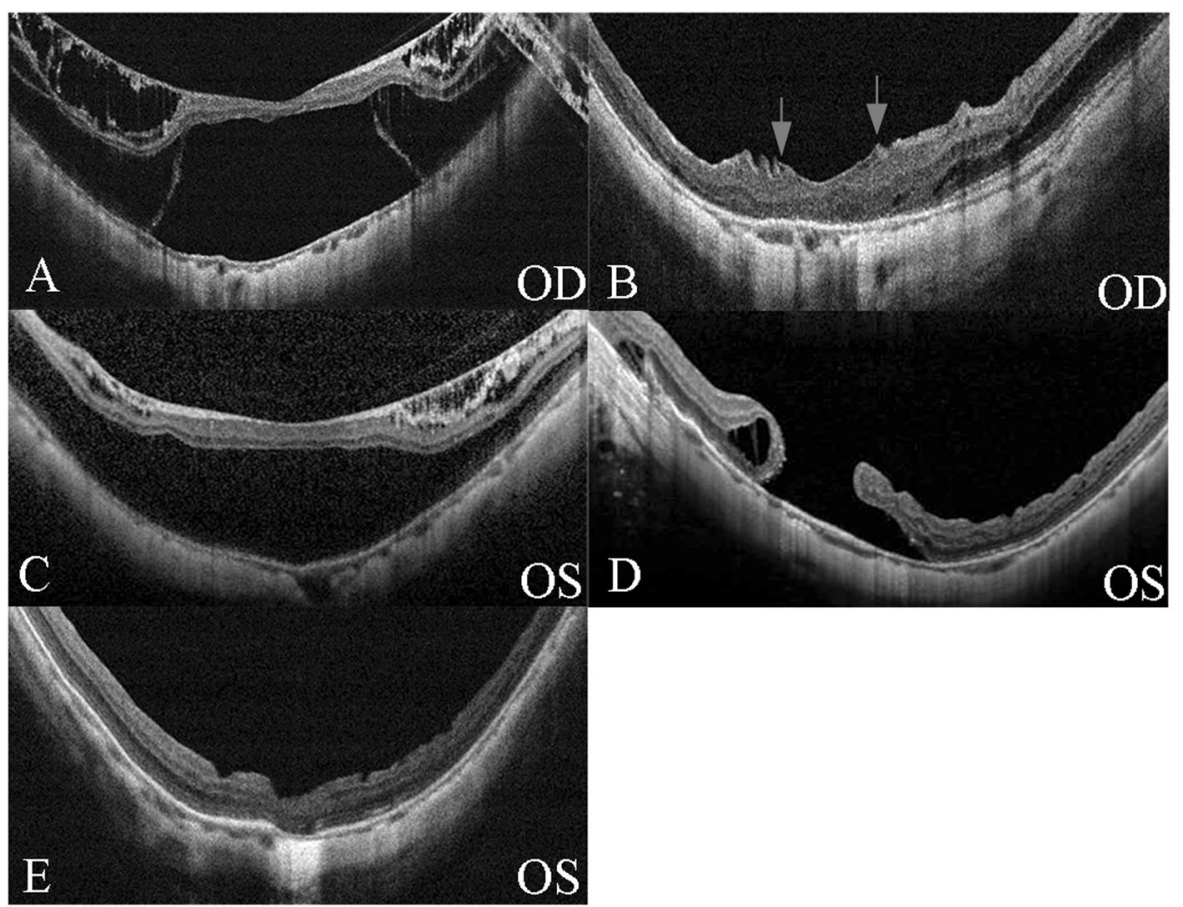

Fig. 3 Optical coherence tomography (OCT) images of one patient who underwent fovea-sparing internal limiting membrane (ILM) and total ILM peeling for two eyes, respectively. a Preoperative OCT image of the right eye with axial length of $28.32 \mathrm{~mm}$. The inner and outer layer of retinoschisis with foveal retinal detachment (RD) can be observed. The preoperative best-corrected visual acuity (BCVA) was $1.5 \operatorname{logMAR}$. b Three months after fovea-sparing ILM peeling, macular retinoschisis was resolved and the edge of the preserved ILM was detected (red

peeling. In addition, OLMH might be enlarged and the thin foveal retina was stretched over a larger arc created by the surgical retinal reattachment, resulting in the development of postoperative FTMH. Thus, we suggested that OLMH might be another risk factor of postoperative FTMH, no matter whether in the FS group or the TP group. The rate of complete resolution of macular schisis was higher in the FS group than in the TP group $(P=0.04)$; however, the mechanisms remain unclear. Based on a review of the ultrastructure, Gass [19] believed that Müller cell cones supply structural support to the fovea. Thus, we hypothesized that the Müller cells endfeet within the preserved ILM may contribute to maintaining the foveolar cone skeleton. In the current study, no contraction of the retained ILM was observed in OCT scanning. We considered that the retained ILM is so thin and small that it could not generate centrifugal tangential traction after vitrectomy. Theoretically, total ILM peeling relieves all tangential traction. However, total ILM peeling can generate potential trauma to the underlying Müller cells and weaken the macular glial structure [20].

In Shimada's study [7], patients in the fovea-sparing group had a better visual improvement than complete arrow). The postopertative BCVA was $0.9 \log$ MAR. $\mathbf{c}$ Preoperative OCT image of the left eye with an axial length of $29.27 \mathrm{~mm}$. OCT showed macular retinoschisis with foveolar RD. The preoperative BCVA was $0.83 \log$ MAR. d At 1 month after total ILM peeling, a full-thickness macular hole $(\mathrm{MH})$ with RD has developed. e Autologous lens capsular transplantation was performed and $\mathrm{MH}$ with RD was resolved. The BCVA improved to $0.5 \log$ MAR

peeling group, which was consistent with both Ho's study [9] and the present study's results. However, the BCVA change was not significant or even progressively decreased in the total peeling group in their studies. In the current study, the BCVA improved significantly both in the FS group and the TP group at the last follow-up (20.9 months vs. 19.6 months). We believe that the visual improvement might be attributed to the restoration of the foveal schisis and retinal reattachment, no matter whether in the FS group or TP group.

To date, there is no consensus on the criteria for the selection of the surgical procedure to treat macular schisis with high myopia. Additionally, it is extremely challenging to preserve the epi-foveal ILM, especially for highly myopic eyes with long ALs, which suggests that further studies to clarify the criteria for appropriate surgery and more practical techniques are needed.

In conclusion, modified fovea-sparing ILM peeling has achieved better resolution of macular retinoschisis and visual improvement. Preoperative OLMH can be a risk factor for the development of postoperative FTMH. The optimal size of the preserved ILM and safety of foveasparing ILM peeling requires further study. 


\section{Summary}

\section{What was known before}

- The risk of FTMH is high in myopic foveoschisis eyes undergoing total ILM peeling.

- Fovea-sparing ILM peeling contributes to better anatomical and visual results than total peeling.

\section{What this study adds}

- FTMH could occur in eyes undergoing fovea-sparing ILM peeling.

- In addition to foveal retinal detachment, OLMH may be another new risk factor for developing postoperative FTMH in myopic foveoschisis.

Acknowledgements We thank the participating patients and the medical staff at Xinhua Hospital Affiliated to Shanghai Jiao Tong University School of Medicine.

Funding The study was supported in part by grants from the National Natural Science Foundation of China (81470642) and the Science and Technology Commission of Shanghai Municipality, China (15XD1502800).

\section{Compliance with ethical standards}

Conflict of interest The authors declare that they have no conflict of interest.

\section{References}

1. Takano M, Kishi S. Foveal retinoschisis and retinal detachment in severely myopic eyes with posterior staphyloma. Am J Ophthalmol. 1999;128(4):472-6.

2. Baba T, Ohno-Matsui K, Futagami S, Yoshida T, Yasuzumi K, Kojima A, et al. Prevalence and characteristics of foveal retinal detachment without macular hole in high myopia. Am J Ophthalmol. 2003;135(3):338-42.

3. Gaucher D, Haouchine B, Tadayoni R, Massin P, Erginay A, Benhamou N, et al. Long-term follow-up of high myopic foveoschisis: natural course and surgical outcome. Am J Ophthalmol. 2007;143(3):455-62.

4. Kanda S, Uemura A, Sakamoto Y, Kita H. Vitrectomy with internal limiting membrane peeling for macular retinoschisis and retinal detachment without macular hole in highly myopic eyes. Am J Ophthalmol. 2003;136(1):177-80.

5. Ikuno Y, Sayanagi K, Ohji M, Kamei M, Gomi F, Harino S, et al. Vitrectomy and internal limiting membrane peeling for myopic foveoschisis. Am J Ophthalmol. 2004;137(4):719-24.

6. Johnson MW. Myopic traction maculopathy: pathogenic mechanisms and surgical treatment. Retina. 2012;32 Suppl 2: S205-10.

7. Shimada N, Sugamoto Y, Ogawa M, Takase H, Ohno-Matsui K. Fovea-sparing internal limiting membrane peeling for myopic traction maculopathy. Am J Ophthalmol. 2012;154(4):693-701.

8. Panozzo G, Mercanti A. Vitrectomy for myopic traction maculopathy. Arch Ophthalmol. 2007;125(6):767-72.

9. Ho TC, Yang CM, Huang JS, Yang CH, Yeh PT, Chen TC, et al. Long-term outcome of foveolar internal limiting membrane nonpeeling for myopic traction maculopathy. Retina. 2014;34 (9):1833-40.

10. Tano Y. Pathologic myopia: where are we now? Am J Ophthalmol. 2002;134(5):645-60.

11. Benhamou N, Massin P, Haouchine B, Erginay A, Gaudric A. Macular retinoschisis in highly myopic eyes. Am J Ophthalmol. 2002;133(6):794-800.

12. Shimada N, Ohno-Matsui K, Baba T, Futagami S, Tokoro T, Mochizuki M. Natural course of macular retinoschisis in highly myopic eyes without macular hole or retinal detachment. Am J Ophthalmol. 2006;142(3):497-500.

13. Todorich B, Scott IU, Flynn HW Jr., Chang S. Macular retinoschisis associated with pathologic myopia. Retina. 2013;33 (4):678-83.

14. Ho TC, Yang CM, Huang JS, Yang CH, Chen MS. Foveola nonpeeling internal limiting membrane surgery to prevent inner retinal damages in early stage 2 idiopathic macula hole. Graefes Arch Clin Exp Ophthalmol. 2014;252(10):1553-60.

15. Ho TC, Chen MS, Huang JS, Shih YF, Ho H, Huang YH. Foveola nonpeeling technique in internal limiting membrane peeling of myopic foveoschisis surgery. Retina. 2012;32(3):631-4.

16. Jin $\mathrm{H}$, Zhang $\mathrm{Q}$, Zhao P. Fovea sparing internal limiting membrane peeling using multiple parafoveal curvilinear peels for myopic foveoschisis: technique and outcome. BMC Ophthalmol. 2016;16(1):180.

17. Hirakata A, Hida T. Vitrectomy for myopic posterior retinoschisis or foveal detachment. Jpn J Ophthalmol. 2006;50(1):53-61.

18. Gao X, Ikuno Y, Fujimoto S, Nishida K. Risk factors for development of full-thickness macular holes after pars plana vitrectomy for myopic foveoschisis. Am J Ophthalmol. 2013;155(6):1021-7 e1.

19. Gass JD. Muller cell cone, an overlooked part of the anatomy of the fovea centralis: hypotheses concerning its role in the pathogenesis of macular hole and foveomacualr retinoschisis. Arch Ophthalmol. 1999;117(6):821-3.

20. Wolf S, Schnurbusch U, Wiedemann P, Grosche J, Reichenbach A, Wolburg H. Peeling of the basal membrane in the human retina: ultrastructural effects. Ophthalmology. 2004;111 (2):238-43 